Draft version December 25, 2013

Preprint typeset using LATEX style emulateapj v. 5/2/11

\title{
DOUBLE DEGENERATE MERGERS AS PROGENITORS OF HIGH-FIELD MAGNETIC WHITE DWARFS
}

\author{
Enrique García-Berro $^{1,2}$, Pablo Lorén-Aguilar ${ }^{1,2}$, Gabriela Aznar-Siguán $^{1,2}$, Santiago Torres ${ }^{1,2}$, \\ Judit Camacho ${ }^{1,2}$, Leandro 'G. Althaus ${ }^{3,4}$, Alejandro H. Córsico ${ }^{3,4}$, Baybars Külebi ${ }^{5,2}$, \& Jordi Isern ${ }^{5,2}$ \\ ${ }^{1}$ Departament de Física Aplicada, Universitat Politècnica de Catalunya, c/Esteve Terrades, 5, 08860 Castelldefels, Spain \\ ${ }^{2}$ Institute for Space Studies of Catalonia, c/Gran Capità 2-4, Edif. Nexus 104, 08034 Barcelona, Spain \\ ${ }^{3}$ Facultad de Ciencias Astronómicas y Geofísicas, Universidad Nacional de La Plata, Paseo del Bosque s/n, (1900) La Plata, Argentina \\ ${ }^{4}$ Instituto de Astrofísica La Plata, IALP (CCT La Plata), CONICET, Argentina \\ ${ }^{5}$ Institut de Ciències de l'Espai (CSIC), Facultat de Ciències, Campus UAB, Torre C5-parell, 08193 Bellaterra, Spain \\ Draft version December 25, 2013
}

\begin{abstract}
High-field magnetic white dwarfs have been long suspected to be the result of stellar mergers. However, the nature of the coalescing stars and the precise mechanism that produces the magnetic field are still unknown. Here we show that the hot, convective, differentially rotating corona present in the outer layers of the remnant of the merger of two degenerate cores is able to produce magnetic fields of the required strength that do not decay for long timescales. We also show, using an state-of-the-art Monte Carlo simulator, that the expected number of high-field magnetic white dwarfs produced in this way is consistent with that found in the Solar neighborhood.
\end{abstract}

Subject headings: stars: interiors — stars: evolution — stars: white dwarfs — stars: rotation — stars: magnetic field — stars: binaries

\section{INTRODUCTION}

The merger of two white dwarfs has received considerable interest during the last years because it is thought to be at the origin of several interesting astrophysical phenomena. In particular, the coalescence of two white dwarfs is one of the possible scenarios - the so-called double-degenerate scenario - to account for Type Ia supernova outbursts (Webbink 1984; Iben \& Tutukov 1984). Moreover, it is thought as well that the merger of two degenerate cores could lead to the formation of magnetars (King, Pringle \& Wickramasinghe 2001). Also, three hot and massive white dwarfs members of the Galactic halo could be the result of the coalescence of a double white-dwarf binary system (Schmidt et al. 1992; Segretain et al. 1997). Additionally, hydrogendeficient carbon and R Corona Borealis stars (Izzard et al. 2007; Clayton et al. 2007; Longland et al. 2011) are thought to be the consequence of the merging of two white dwarfs. Also, the large metal abundances found around some hydrogen-rich white dwarfs with dusty disks around them could also be explained by the merger of a carbon-oxygen and a helium white dwarf (García-Berro et al. 2007). Last but not least, the phase previous to the coalescence of a double white-dwarf close binary system has been shown to be a powerful source of gravitational waves that would be eventually detectable by LISA (Lorén-Aguilar et al. 2005). Here we show that the merger of two degenerate cores can also explain the presence of very high magnetic fields in some white dwarfs - a result previously anticipated by Wickramasinghe \& Ferrario (2000) but not yet quantitatively proved.

High-field magnetic white dwarfs have magnetic fields in excess of $10^{6} \mathrm{G}$ and up to $10^{9} \mathrm{G}$ (Schmidt et al. 2003). Surprisingly, very few belong to a non-interacting binary system (Kawka et al. 2007), and moreover they are more massive than average (Silvestri et al. 2007). One pos-

garcia@fa.upc.edu sibility is that these white dwarfs descend from single stars, so the magnetic field is a fossil of previous evolution (Angel et al. 1981). However, this scenario cannot explain why these stars are massive, and why they are not found in non-interacting binary systems. Recently, it has been suggested (Tout et al. 2008) that strong magnetic fields are produced during a common envelope episode in a close binary system in which one of the components is degenerate. During this phase, spiral-in of the secondary induces differential rotation in the extended convective envelope, resulting in a stellar dynamo which produces the magnetic field. However, the magnetic field produced in this way does not penetrate in the white dwarf, and decays rapidly when the common envelope is ejected (Potter \& Tout 2010).

In this paper we show that the hot, differentially rotating, convective corona resulting from the merger of two degenerate cores produces strong magnetic fields, which are confined to the outer layers of the resulting remnant, and which do not decay for very long timescales. The paper is organized as follows. In Sect. 2 we explain the precise mechanism that produces the required magnetic fields, and we show that these fields are confined the outer layers of the remnant of the coalescence and do not decay for very long timescales. Sect. 3 is devoted to analyze if our model can account for the number of high-field magnetic white dwarfs in the Solar neighborhood, while in Sect. 4 we summarize our findings and we present our conclusions.

\section{THE STELLAR DYNAMO}

The merger of two degenerate cores is the final destiny of the evolution of a sizable fraction of binary systems. Three-dimensional simulations of the merger process (Guerrero et al. 2004; Yoon et al. 2007; LorénAguilar et al. 2009) indicate that the remnant of the coalescence of two white dwarfs consists of a central white dwarf which contains all the mass of the primary. On top 
of it a hot corona, which is made of approximately half of the mass of the disrupted secondary, can be found. Finally, surrounding this compact remnant a rapidly rotating Keplerian disk is formed, containing nearly all the mass of the secondary which has not been incorporated to the hot corona. According to these calculations little mass $\left(\sim 10^{-3} M_{\odot}\right)$ is ejected from the system during the merger. The structure of the remnant of the coalescence is illustrated in Fig. 1.

The existing simulations show that the temperature gradient in the hot corona is high, and consequently the corona is convective. We computed the boundaries of the convective region using the Schwarzschild criterion, and we found that the inner and outer edges of the convective region are located at radii $R \approx 0.012 R_{\odot}$, and $R \approx 0.026 R_{\odot}$, respectively, and that the total mass inside this region is $\sim 0.24 M_{\odot}$ (see Fig. 1). Moreover, this region rotates differentially, and is prone to magnetorotational instability. Assuming energy equipartition, the resulting $\alpha \omega$ dynamo produces a magnetic field $B^{2} / 8 \pi \approx \rho(\omega R)^{2} / 2$. For the typical values found in the simulations of Lorén-Aguilar et al. (2009), the magnetic field amounts to $B \sim 3.2 \times 10^{10} \mathrm{G}$. Thus, the energy available in the convective corona is sufficiently large to produce strong magnetic fields. We note that even in the case in which only $0.1 \%$ of the total energy of the convective shell is invested in magnetic energy the fields produced in this way are of the order of $10^{7} \mathrm{G}$, a value typical of high-field magnetic white dwarfs. We also note that there are very few white dwarfs with magnetic fields larger than $10^{9} \mathrm{G}$, and that when these fields are observed, these are confined to spots on their surfaces.

For this mechanism to be efficient at producing the observed magnetic fields, the dynamo must work for several convective turnovers before the energy of the hot corona is radiated away. The temperature of the corona is so high that is preferentially cooled by neutrino emission. The neutrino luminosity of the corona is $L_{\nu} \sim$ $4.0 \times 10^{2} L_{\odot}$, while the total thermal energy of the nondegenerate material in the corona is $U \sim 8.8 \times 10^{48} \mathrm{erg}$. Hence, the convective shell lasts for $\tau_{\text {hot }} \sim 1.8 \times 10^{5} \mathrm{yr}$. The convective turnover timescale is $\tau_{\text {conv }} \approx H_{P} / v_{\text {conv }}$, where $H_{P} \approx 2.7 \times 10^{8} \mathrm{~cm}$ is the pressure scale height and $v_{\text {conv }} \approx 8.0 \times 10^{7} \mathrm{~cm} / \mathrm{s}$ is the convective velocity. Thus, $\tau_{\text {conv }} \sim 3.3 \mathrm{~s}$, and during the lifetime of the hot corona the number of convective cycles is sufficiently large. Consequently, the $\alpha \omega$ mechanism is able to produce a strong magnetic field. We also note that if the duration of the convective shell is substantially smaller than that estimated here, large magnetic fields can still be produced. Specifically, even assuming durations $10^{3}$ times smaller than that previously estimated, the number of convective cycles would still be enough to produce magnetic fields comparable to those observationally found in high-field magnetic white dwarfs. We thus conclude that the stellar dynamos produced in the aftermath of the merger of two degenerate cores can produce magnetic fields of the order of $10^{7}$ G. From now on we adopt this value (typical of high-field magnetic white dwarfs) as a fiducial value for the rest of the calculations.

Once the magnetic field is established we need to know if it can diffuse outwards, to the surrounding disk, or inwards, to the degenerate primary. To this end we

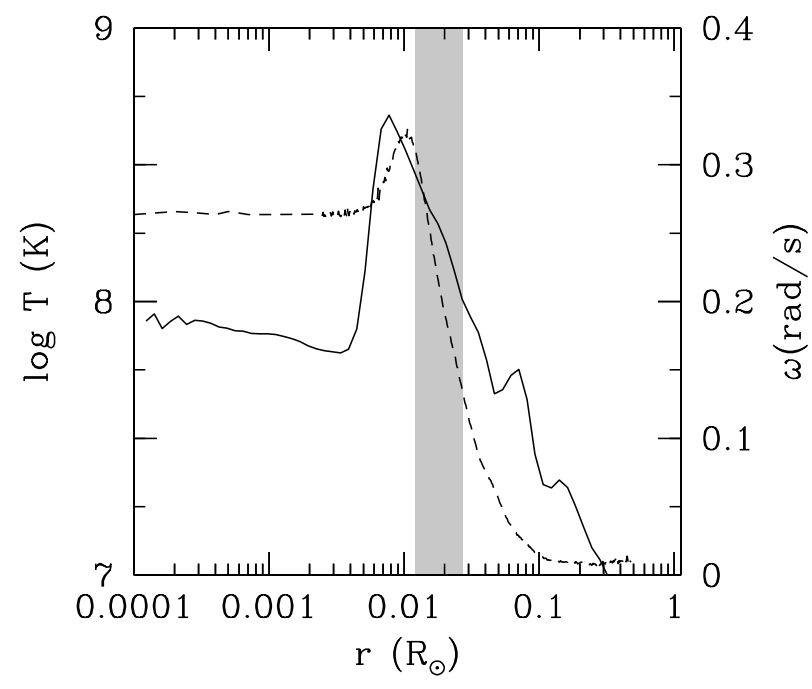

FIG. 1.- Dynamo configuration in a white dwarf merger. Temperature (solid line, left scale) and rotational velocity (dashed line, right scale) profiles of the final remnant of a white dwarf merger as a function of radius are shown for the case of a binary system composed of two stars of 0.6 and $0.8 M_{\odot}$. In our simulations approximately half of the mass of the disrupted secondary $\left(\sim 0.3 M_{\odot}\right)$ is accreted onto the primary, while the rest of the mass goes to form the Keplerian disk, which extends up to $\sim 0.3 R_{\odot}$. The temperature of the central white dwarf depends on the initial temperature of the coalescing white dwarfs (Yoon et al. 2007; Lorén-Aguilar et al. 2009). However, the temperature of the hot corona is remarkably similar in all the simulations, $T \approx 8.7 \times 10^{8} \mathrm{~K}$. The central spinning white dwarf rotates as a rigid body with a rotational velocity $\omega \sim 0.26 \mathrm{~s}^{-1}$, and the corona rotates differentially, with a peak rotation rate $\omega \sim 0.33 \mathrm{~s}^{-1}$. These velocities arise from energy and angular momentum conservation, since little mass is ejected from the system, so the orbital angular momentum of the binary system is invested in spinning up the remnant, while the available energy is primarily invested in heating the corona. The location of the convective region is displayed by the shaded area.

solved the diffusion equation (Jackson 1998; Wendell et al. 1987):

$$
\frac{\partial \vec{B}}{\partial t}=-\vec{\nabla} \times\left(\frac{c^{2}}{4 \pi \sigma} \vec{\nabla} \times \vec{B}-\vec{v} \times \vec{B}\right)
$$

being $\sigma$ the magnetic conductivity, for which we used the most up-to-date prescription (Cassisi et al. 2007), while the rest of the symbols have their usual meaning. We first integrated the diffusion equation for the surrounding disk using cylindrical coordinates, adopting the velocity field and density and temperature profiles resulting from our SPH simulations (Lorén-Aguilar et al. 2009). The initial condition adopted here is $\vec{B}(0)=\overrightarrow{0}$, while we also imposed the boundary condition $\partial \vec{B} / \partial t=0$ at the outer edge of the disk. We used a Crank-Nicolson integration scheme with variable coefficients (Dautray \& Lions 2000) which turns out to be stable. We found that the timescale for diffusion of the magnetic field across the disk is $\tau_{\text {disk }} \sim 2.0 \times 10^{11} \mathrm{yr}$. We did the same calculation, this time using spherical coordinates, to estimate the penetration of the magnetic field in the dense, degenerate primary. The use of spherical coordinates is perfectly justified because the departures of the compact primary from sphericity are very small. For this 
calculation we followed the procedure outlined in Wendell et al. (1987). In this case the electrical conductivity is totally dominated by degenerate electrons, and depends on the adopted temperature of the isothermal white dwarf. If a temperature $T \approx 7.6 \times 10^{7} \mathrm{~K}$ is taken (Lorén-Aguilar et al. 2009) the diffusion timescale turns out to be $\tau_{\mathrm{WD}} \sim 4.3 \times 10^{9} \mathrm{yr}$. Since this timescale depends on the adopted initial temperature we computed, using an up-to-date stellar evolutionary code (Renedo et al. 2010), a cooling sequence for a white dwarf of the mass, $1.1 M_{\odot}$, and chemical composition of the remnant, a carbon-oxygen core. The time to cool from $7.6 \times 10^{7} \mathrm{~K}$ to a value typical of field white dwarfs (say $3.0 \times 10^{6} \mathrm{~K}$ ) is very short, $\tau_{\text {cool }} \sim 3.0 \times 10^{7}$ yr. Hence, we can safely assume that as the white dwarf cools, the rapid increase of the electrical conductivity does not allow the magnetic field to penetrate in the degenerate core of the primary, and remains confined to the surface layers.

Our model naturally predicts that the masses of highfield magnetic white dwarfs should be larger than average and that they should be observed as single white dwarfs, as observationally found - see, for instance, Valyavin \& Fabrika (1999). However, high-field magnetic white dwarfs are generally found to be slow rotators (Wickramasinghe \& Ferrario 2000). This apparent contradiction of the model can be easily solved. If the rotation and magnetic axes are misaligned, magneto-dipole radiation rapidly spins down the white dwarf - see, however, Timokhin (2006) and Spitkovsky (2006), where the time evolution of magnetospheres for axisymmetric and oblique rotators is described in detail. The evolution of the rotational velocity when both axes are misaligned (Benacquista et al. 2003) is:

$$
\dot{\omega}=-\frac{2 \mu^{2} \omega^{3}}{3 I c^{3}} \sin ^{2} \alpha
$$

where $I$ is the moment of inertia of the white dwarf, $\alpha$ is the angle between the magnetic and rotation axes and $\mu=B R_{\mathrm{WD}}^{3}$. Thus, the spin-down timescale is simply $\tau_{\mathrm{MDR}}=\omega / 2 \dot{\omega}$. Adopting the values resulting from our SPH simulations (Lorén-Aguilar et al. 2009) we obtain $\tau_{\mathrm{MDR}} \sim 2.4 \times 10^{8} / \sin ^{2} \alpha$ yr, for a field strength $B=10^{7} \mathrm{G}$. Hence, if both axes are perfectly aligned the remnant of the coalescence will be a high-field, rapidly rotating magnetic white dwarf. On the contrary, if both axes are nearly perpendicular magneto-dipole radiation efficiently brakes the remnant. Consequently, the very young, hot, ultramassive, slowly rotating magnetic white dwarfs 1RXS J0823.6-2525 and PG 1658+441, which have fields $\sim 3.5 \times 10^{6} \mathrm{G}$ can be accommodated in our model. For instance, PG 1658+441 has an effective temperature $T_{\text {eff }} \sim 30000 \mathrm{~K}$ and a mass $M \sim 1.3 M_{\odot}$ (Dupuis et al. 2003), which corresponds to a cooling age of $\sim 3.7 \times 10^{8} \mathrm{yr}$, while the time needed to brake the white dwarf equals to $\sim 8.4 \times 10^{7} \mathrm{yr}$ if $\sin \alpha=1$ is adopted. Thus, our model can account for the slow rotation rate of $\mathrm{PG} 1658+441$, provided that the rotation and magnetic axes are misaligned. We also note at this point that given the small radii - or, equivalently, the small radiating surfaces - of massive white dwarfs, their cooling ages increase markedly for effective temperatures smaller than $\log T_{\text {eff }} \lesssim 4.7$.

On the other hand, rapidly rotating magnetic white dwarfs - of which an example is RE J 0317-853, a very massive $\left(\sim 1.3 M_{\odot}\right)$ white dwarf belonging to a wide binary system (Külebi et al. 2010) - could be the result of nearly equal-mass mergers in which both axes are aligned. In this case no Keplerian disk is formed, so these white dwarfs will not show infrared excesses. If, on the contrary, the masses of the coalescing white dwarfs are different, the axes are not aligned, and the disk is able to survive for long enough times, a second-generation planetary system could be eventually formed (GarcíaBerro et al. 2007) and tidal disruption and accretion of minor planets may contaminate the atmosphere of the white dwarf, resulting in magnetic DAZ or DAZd white dwarfs possibly showing infrared excesses. Examples of such white dwarfs are NLTT 10480 (Kawka \& Vennes 2011) and G77-5018 (Farihi et al. 2011), for which a satisfactory explanation is still lacking.

The geometry of the surface magnetic fields of white dwarfs has been investigated over the years using spectropolarimetric observations. The available wealth of observations shows that, in almost all cases, magnetic white dwarfs have fields deviating strongly from dipolar structure. In particular, it appears that in most cases the geometry of the surface magnetic field corresponds to quadrupolar or higher multipoles - e.g., PG 1031+234 (Schmidt et al. 1986), REJ 0317-855 (Ferrario 1997), HE 0241-0155 (Reimers et al. 2005), HE 1045-0908 (Euchner et al. 2005), or PG 1015+014 (Euchner et al. 2006). This means that the toroidal component of the magnetic field must be stable, and that a poloidal component should also be present. Our mechanism can also qualitatively reproduce these observations. Indeed, in the $\alpha \omega$ mechanism, convection is responsible for the generation of poloidal fields, whereas rotation is responsible for the generation of toroidal fields. Specifically, the energy available to generate the poloidal field component is $\rho v_{\text {conv }}^{2} / 2 \sim 4.0 \times 10^{20} \mathrm{erg}$, which is $\sim 10 \%$ of the energy available to build the toroidal component, $\rho(\omega R)^{2} / 2 \sim 5.5 \times 10^{21} \mathrm{erg}$. Thus, we expect that the magnetic field generated by the fast rotating convective shell will have both toroidal and poloidal field components. Moreover, it is well known that purely toroidal fields are unstable due to Tayler (1973) instability. However, the existence of a small poloidal component is a sufficient condition for stability. Actually, recent numerical studies (Braithwaite 2009) show that the energy stored in the poloidal field component can be as high as $80 \%$ of the total magnetic energy, and that even poloidal components $10^{-6}$ times smaller than the toroidal one are enough to warrant stability. Thus, toroidal fields generated by the rotating, convective shell produced in the merger of two double degenerates are stable, and moreover we expect that the magnetic fields generated in this way will have a complex geometry, in agreement with observations.

\section{MAGNETIC WHITE DWARFS IN THE SOLAR NEIGHBORHOOD}

To assess if our scenario can reproduce the observed number of high-field magnetic white dwarfs we have expanded an existing, state-of-the-art Monte Carlo code (García-Berro et al. 1999; Torres et al. 2002; GarcíaBerro et al. 2004) designed to study the Galactic populations of single white dwarfs to deal with those of double 
degenerates. In our simulations we assumed that $50 \%$ of stars belong to binaries, and we normalized to the local disk density (Holmberg \& Flynn 2000). The initial primary masses were obtained using a standard initial mass function (Kroupa et al. 1993), and the initial mass ratios according to a flat distribution. Also, a constant star formation rate and a disk age of $10^{10} \mathrm{yr}$ were adopted. Orbital separations and eccentricities were randomly drawn according to a logarithmic probability distribution (Nelemans et al. 2001) and to a thermal distribution (Heggie 1975), respectively. For each of the components of the binary analytical fits to detailed stellar evolutionary tracks were used (Hurley et al. 2000), which provide full coverage from the main sequence until advances stages of evolution. For the white dwarf stage the most recent cooling tracks of Renedo et al. (2010) were employed. The orbital evolution of each binary was computed taking into account circularization and synchronization (Hurley et al. 2002). We also considered mass losses through stellar winds. Specifically, we assumed that the evolution during the main sequence is conservative, and only after it we included stellar winds. The adopted mass-loss rate is that of Kudritzki \& Reimers (1978) except on the AGB, for which the rate of Vassiliadis \& Wood (1993) was used. Angular momentum losses due to magnetic braking and gravitational radiation were also included (Schreiber et al. 2003; Zorotovic et al. 2010). The Roche lobe radius was computed using the most commonly used approximation (Eggleton 1983), and during the overflow episodes both rejuvenation and ageing were taken into account (Hurley et al. 2002). For the common envelope phase we considered standard prescriptions for the common envelope efficiency and for the fraction of gravitational binding energy of the donor available to eject the envelope - see, e.g., Dewi \& Tauris (2000). Specifically, we adopted $\alpha_{\mathrm{CE}}=0.25$ and a variable value for the binding energy parameter $\lambda$ (Zorotovic et al. 2010). With all these ingredients we found that the fraction of merged double degenerate cores in the solar neighborhood is $\sim 2.9 \%$ of the total synthetic population. This number includes not only white dwarf mergers $(\sim 0.3 \%)$, but also the coalescence of a white dwarf and a giant star with a degenerate core $(\sim 1.1 \%)$, and the merger of two giants with degenerate cores $(\sim 1.5 \%)$. In these two last cases - namely, the merger of a white dwarf and a giant, and the merger of two giant stars - the coalescences occur during the common envelope phase, while the merger of two white dwarfs is driven by gravitational wave radiation. Finally, we emphasize that the number of white dwarf mergers we obtain is in line with those obtained using completely different approaches (Bogomazov \& Tutukov 2009) — see below for a detailed comparison with our results for the solar neighborhood - and that the distribution of remnant masses is nearly flat, in accordance with the observed distribution of masses of magnetic white dwarfs (Należyty \& Madej 2004).

To better illustrate this last issue, Fig. 2 shows the frequency distribution of remnant masses of the different merger channels for a sample of $10^{3}$ mergers. We assumed that the remnant of each coalescence has a mass $M=M_{1}+M_{2} / 2$, where $M_{1}$ and $M_{2}$ stand, respectively, for the masses of the primary and secondary degenerate cores. This choice is in accordance with the results of the SPH simulations of Lorén-Aguilar et al. (2009),

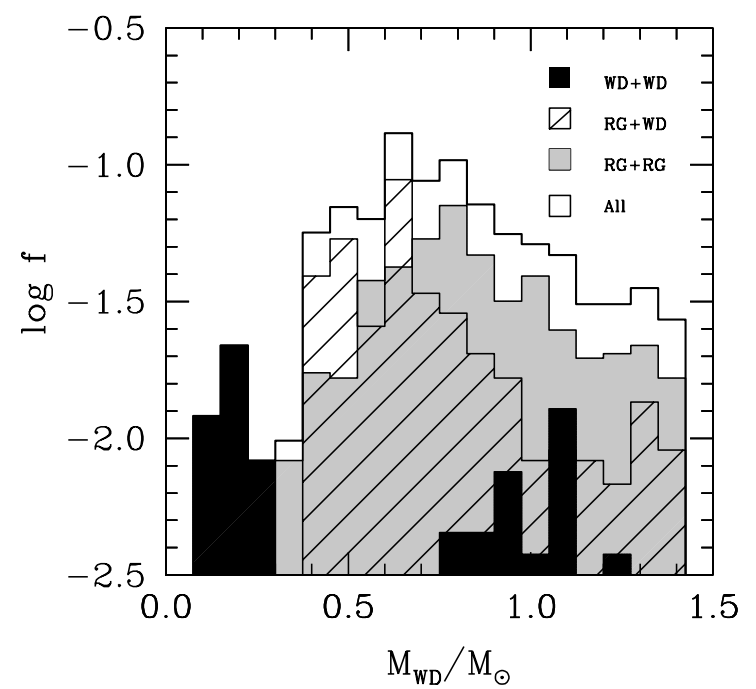

FIG. 2.- Mass distribution of the remnants of the mergers. The distribution shows the frequency of the different merger channels. The black histogram shows the masses of the remnants of the mergers of double white dwarf binaries, the dashed histogram that of the mergers of a binary system composed of a red giant and a white dwarf, the shaded histogram that of the mergers of two red giants, while the total mass distribution is shown using a solid line.

which show that approximately half of the secondary is accreted onto the primary, while the rest of the mass of the disrupted secondary forms a debris region and little mass is ejected during the merger. We also eliminated all those remnants that have masses larger than Chandrasekhar's limit, while for the mergers producing a helium white dwarf we adopted the procedure of Hurley et al. (2002). As can be seen, the total mass distribution (open histogram) presents a first peak for masses smaller than $\sim 0.4 M_{\odot}$, corresponding to mergers in which a helium white dwarf is produced, then sharply increases for increasing remnant masses and afterwards smoothly decreases for masses larger than $\sim 0.6 M_{\odot}$. When this distribution is sampled for $\sim 26$ objects with masses ranging from $0.8 M_{\odot}$ to $1.4 M_{\odot}$ we usually obtain fairly flat distributions, although the scarce number of objects and the subsequent large deviations prevent a sound comparison with the observational data.

Within $20 \mathrm{pc}$ of the Sun there are 122 white dwarfs (Holberg et al. 2008), and several of them are magnetic (Kawka et al. 2007). This sample is $80 \%$ complete, but subject to poor statistics. However, it is useful because for it we have a reliable determination of the true incidence of magnetism in white dwarfs. Mass determinations are available for 121 of them. In the local sample there are 14 magnetic white dwarfs, 8 have magnetic fields in excess of $10^{7} \mathrm{G}$, and 3 have masses larger than $0.8 M_{\odot}$ - a value which is $\sim 2.5 \sigma$ away from the average mass of field white dwarfs. The selection of this mass cut is somewhat arbitrary but, given the strong bias introduced by the initial mass function, we expect that the vast majority of high-field magnetic white dwarfs more massive than $0.8 M_{\odot}$ would be the result of stellar mergers. This is indeed the case, since our population synthesis calculations predict that $\sim 4$ white dwarfs are the result of double degenerate mergers, and 
have masses larger than $0.8 M_{\odot}$, in good agreement with observations. Additionally, our simulations predict that the fraction of white dwarfs more massive than $\sim 0.8 M_{\odot}$ resulting from single stellar evolution is $\sim 10 \%$. Consequently, the expected number of massive white dwarfs in the local sample should be $\sim 12$. Instead, the local sample contains 20, pointing towards a considerable excess of massive white dwarfs, which could be the progeny of mergers. The rest of the population of magnetic white dwarfs $(\sim 5)$ would be well the result of the evolution of single stars (Aznar Cuadrado et al. 2004). In this case the magnetic field could have been generated during the red giant phase (Blackman et al. 2001), or could be a fossil of the evolution of magnetic Ap or Bp stars - see, for instance, Mathys et al. (2001) and references therein. Note, nevertheless, that it has been recently suggested that these massive main-sequence stars are also the result of stellar mergers (Tutukov \& Fedorova 2010).

The number of coalescing binaries previously discussed compares well with the results obtained using very different population synthesis codes. For instance, Bogomazov \& Tutukov (2009), using a completely independent code, obtain $\sim 7 \pm 1$, where we have assumed poissonian errors, white dwarf mergers within $20 \mathrm{pc}$ of the Sun see their Table 1 - whereas we obtain $\sim 4 \pm 2$. Thus, within the sampling uncertainties, both numbers agree qualitatively, despite the very small number of objects and the existing theoretical uncertainties, which include, among others, the completely different approaches used to simulate a representative sample of binaries, the ingredients necessary to model the Galaxy as a whole, and the parameters adopted in the calculation of the common envelope phase.

\section{SUMMARY AND CONCLUSIONS}

We have shown that the hot, convective, differentially rotating corona predicted by detailed three-dimensional simulations of the merger of two degenerate stellar cores is able to produce very high magnetic fields. We have also shown that these magnetic fields are confined to the outer layers of the remnant of the coalescence, and do not propagate neither to the interior of the white dwarf or to the debris region. Our model naturally predicts that high-field magnetic white dwarfs should preferentially have high masses, and should be observed as single white dwarfs, as observationally found. Moreover, if the rotation and magnetic axes are not aligned magnetodipole radiation rapidly spins down the magnetic white dwarf in short timescales. Thus, high-field magnetic white dwarfs may have a large variety of rotation periods. Moreover, in the case in which the masses of the coalescing white dwarfs are not equal the heavy rotationally supported disk can survive for long periods of time, and a planetary system could eventually form. Disruption of small planets could then contaminate the very outer layers of the magnetic white dwarf, explaining the recently discovered population of metallic magnetic white dwarfs. If, on the contrary, the masses of the merging white dwarfs are similar the remnant has spherical symmetry and rotates very rapidly, as observed in some high-field magnetic white dwarfs. Also, the geometry of the surface magnetic fields can be well explained by our model. Finally, we have also shown that the expected number of double degenerate mergers is roughly consistent with the number of high-field magnetic white dwarfs in the local sample. In summary, our calculations indicate that a sizable fraction of all high-field magnetic white dwarfs could be the result of double degenerate mergers, in accordance with previous suggestions (Wickramasinghe \& Ferrario 2000) not hitherto proven.

This research was supported by AGAUR, by MCINN grants AYA2011-23102 and AYA08-1839/ESP, by the European Union FEDER funds, by the ESF EUROGENESIS project (grant EUI2009-04167), by AGENCIA: Programa de Modernización Tecnológica BID 1728/OCAR, and by PIP 2008-00940 from CONICET.

\section{REFERENCES}

Angel, J. R. P., Borra, E. F., \& Landstreet, J. D. 1981, ApJS, 45, 457

Aznar Cuadrado, R., Jordan, S., Napiwotzki, R., et al. 2004, A\&A, 423, 1081

Benacquista, M., Sedrakian, D. M., Hairapetyan, M. V., Shahabasyan, K. M., \& Sadoyan, A. A. 2003, ApJ, 596, L223

Blackman, E. G., Frank, A., Markiel, J. A., Thomas, J. H., \& Van Horn, H. M. 2001, Nature, 409, 485

Bogomazov, A. I., \& Tutukov, A. V. 2009, Astronomy Reports, 53, 214

Braithwaite, J. 2009, MNRAS, 397, 763

Cassisi, S., Potekhin, A. Y., Pietrinferni, A., Catelan, M., \& Salaris, M. 2007, ApJ, 661, 1094

Clayton, G. C., Geballe, T. R., Herwig, F., Fryer, C., \& Asplund, M. 2007, ApJ, 662, 1220

Dautray, R., \& Lions, J.L. 2000, "Mathematical Analysis and Numerical Methods for Science and Technology", vol. 6 (Heidelberg: Springer Verlag)

Dewi, J. D. M., \& Tauris, T. M. 2000, A\&A, 360, 1043

Dupuis, J., Chayer, P., Vennes, S., Allard, N. F., \& Hébrard, G. 2003, ApJ, 598, 486

Eggleton, P. P. 1983, ApJ, 268, 368

Euchner, F., Reinsch, K., Jordan, S., Beuermann, K., Gänsicke, B. T. 2005, A\&A, 442, 651

Euchner, F., Jordan, S., Beuermann, K., Reinsch, K., Gänsicke, B. T. 2006, A\&A, 451, 671
Farihi, J., Dufour, P., Napiwotzki, R., \& Koester, D. 2011, MNRAS, 413, 2559

Ferrario, L., Vennes, S., Wickramasinghe, D. T., Bailey, J. A., \& Christian, D. J. 1997, MNRAS, 292, 205

García-Berro, E., Torres, S., Isern, J.,\& Burkert, A. 1999, MNRAS, 302, 173

García-Berro, E., Torres, S., Isern, J., \& Burkert, A. 2004, A\&A, 418,53

García-Berro, E., Lorén-Aguilar, P., Pedemonte, A. G., et al. 2007, ApJ, 661, L179

Guerrero, J., García-Berro, E., \& Isern, J. 2004, A\&A, 413, 257

Heggie, D. C. 1975, MNRAS, 173, 729

Holberg, J. B., Sion, E. M., Oswalt, T., et al. 2008, AJ, 135, 1225

Holmberg, J., \& Flynn, C. 2000, MNRAS, 313, 209

Hurley, J. R., Pols, O. R., \& Tout, C. A. 2000, MNRAS, 315, 543

Hurley, J. R., Tout, C. A., \& Pols, O. R. 2002, MNRAS, 329, 897

Iben, I., Jr., \& Tutukov, A. V. 1984, ApJS, 54, 335

Izzard, R. G., Jeffery, C. S., \& Lattanzio, J. 2007, A\&A, 470, 661

Jackson, J. D. 1998, "Classical Electrodynamics", 3rd Edition, (New York: John Wiley \& Sons)

Kawka, A., Vennes, S., Schmidt, G. D., Wickramasinghe, D. T., \& Koch, R. 2007, ApJ, 654, 499

Kawka, A., \& Vennes, S. 2011, A\&A, 532, A7

King, A. R., Pringle, J. E., \& Wickramasinghe, D. T. 2001, MNRAS, 320, L45 
Külebi, B., Jordan, S., Nelan, E., Bastian, U., \& Altmann, M. 2010, A\&A, 524, A36

Kudritzki, R. P., \& Reimers, D. 1978, A\&A, 70, 227

Kroupa, P., Tout, C. A., \& Gilmore, G. 1993, MNRAS, 262, 545

Lorén-Aguilar, P., Guerrero, J., Isern, J., Lobo, J. A., \& García-Berro, E. 2005, MNRAS, 356, 627

Lorén-Aguilar, P., Isern, J., \& García-Berro, E. 2009, A\&A, 500, 1193

Longland, R., Lorén-Aguilar, P., José, J., et al. 2011, ApJ, 737, L34

Mathys, G., Solanki, S. K., \& Wickramasinghe, D. T. 2001, "Magnetic Fields Across the Hertzsprung-Russell Diagram", ASP Conf. Proc. vol. 248 (San Francisco: Astron. Soc. of the Pacific)

Należyty, M., \& Madej, J. 2004, A\&A, 420, 507

Nelemans, G., Yungelson, L. R., Portegies Zwart, S. F., \& Verbunt, F. 2001, A\&A, 365, 491

Potter, A. T., \& Tout, C. A. 2010, MNRAS, 402, 1072

Reimers, D., Jordan, S., \& Christlieb, N. 2004, A\&A, 414, 1105

Renedo, I., Althaus, L. G., Miller Bertolami, M. M., et al. 2010, ApJ, 717, 183

Schmidt, G. D., West, S. C., Liebert, J., Green, R. F., \& Stockman, H. S. 1986, ApJ, 309, 218

Schmidt, G. D., Bergeron, P., Liebert, J., \& Saffer, R. A. 1992, ApJ, 394, 603

Schmidt, G. D., Harris, H. C., Liebert, J., et al. 2003, ApJ, 595, 1101

Schreiber, M. R., \& Gänsicke, B. T. 2003, A\&A, 406, 305
Segretain, L., Chabrier, G., \& Mochkovitch, R. 1997, ApJ, 481, 355

Silvestri, N. M., Lemagie, M. P., Hawley, S. L., et al. 2007, AJ, 134,741

Spitkovsky, A. 2006, ApJ, 648, L51

Tayler, R. J. 1973, MNRAS, 161, 365

Timokhin, A. N. 2006, MNRAS, 368, 1055

Torres, S., García-Berro, E., Burkert, A., \& Isern, J. 2002 , MNRAS, 336, 971

Tout, C. A., Wickramasinghe, D. T., Liebert, J., Ferrario, L., \& Pringle, J. E. 2008, MNRAS, 387, 897

Tutukov, A. V., \& Fedorova, A. V. 2010, Astronomy Reports, 54, 156

Valyavin, G., \& Fabrika, S. 1999, in "Proc. of the 11th European Workshop on White Dwarfs", Eds.: S.E. Solheim \& E.G. Meistas, ASP Conf. Proc. vol. 169, 206 (San Francisco: Astron. Soc. of the Pacific)

Vassiliadis, E., \& Wood, P. R. 1993, ApJ, 413, 641

Webbink, R. F. 1984, ApJ, 277, 355

Wendell, C. E., van Horn, H. M., \& Sargent, D. 1987, ApJ, 313, 284

Wickramasinghe, D. T., \& Ferrario, L. 2000, PASP, 112, 873

Yoon, S.-C., Podsiadlowski, P., \& Rosswog, S. 2007, MNRAS, 380,933

Zorotovic, M., Schreiber, M. R., Gänsicke, B. T., \& Nebot Gómez-Morán, A. 2010, A\&A, 520, A86 